\title{
Footprints in Laser Medicine and Surgery: Beginnings, Present, and Future
}

Ki Uk Song

Brighlans, Inc., Manila, Philippines

Received May 12, 2017

Revised June 6, 2017

Accepted June 6, 2017

\section{Correspondence}

Ki Uk Song

Brighlans, Inc., Heritage Tower, \#1851 Vasquez

Street, Malate, Manila, Philippines

Tel.: +63-2-521-0441

Fax: +63-2-521-0441

E-mail: info.brighlansdagmail.com

(C) Korean Society for Laser Medicine and Surgery

(c) This is an open access article distributed under the terms of the Creative Commons Attribution NonCommercial License (http://creativecommons.org/ licenses/by-nc/4.0) which permits unrestricted noncommercial use, distribution, and reproduction in any medium, provided the original work is properly cited.
Light-based science and technology has been evolving throughout history. One of the most significant advances in light-based science and technology is the laser. Early in its development, the laser offered physically unique and attractive characteristics with much potential to solve the conditions of medicine and surgery, opening many gateways for laser applicability through research by renowned physicians. In collaboration with physicians, the medical industry has continued to develop better and more efficient technological advancements. The laser has now become one of the most advanced medical solutions for a variety clinical cases. Moreover, there are some procedures for which the laser is now mandatory. Despite many challenges that still lie ahead, practitioners and patients alike are thrilled to see even more exciting progress in the fast-expanding field of laser medicine and surgery.

\section{Key words}

Laser; History; Selective photothermolysis; Scanning technology; Fractionated laser 


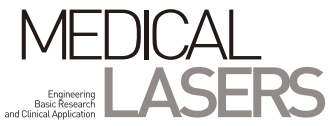

\section{INTRODUCTION}

This science behind the laser was conceptualized from a bare idea and has been continuously developed by many scientists and physicists. Numerous failures in trials have never been able to frustrate progress towards the clinical application of laser energy. Currently, lasers are being used in many specialties in medicine and surgery.

\section{BIRTH OF LASER}

The term laser is an acronym for "Light Amplification by Stimulated Emission of Radiation." The technology emits light through a process of optical amplification based on the stimulated emission of spontaneous electromagnetic radiation in the form of photons. Lasers are used to cut metals in industrial manufacturing, to scan barcodes in commercial stores, to mark targets and measure range and speed in military and law enforcement devices, to emit light displays for entertainment, to burn and to play digital versatile discs (DVDs), and to treat an increasing variety of diseases and conditions. The lasers indicated in today's medicine and surgeries are being used in many specialties, including angioplasty, cancer diagnosis, cancer treatment, frenectomy, lithotripsy, laser mammography, medical imaging, microscopy, ophthalmology, optical coherence tomography, prostatectomy, plastic surgery, laser liposuction, surgery, and cosmetic procedures, such as scar revision, skin resurfacing, vascular lesion treatment, laser hair removal, tattoo removal, and many more.

The theoretical concept behind the laser was originally established by Albert Einstein (1879-1955) in 1917. Einstein proposed the concepts and principles of spontaneous and stimulated emission of radiation after experiments that identified a sudden burst of coherent light as atoms discharged in a rapid chain reaction called "stimulated emission." The first working laser was built in 1960 by Theodore H. Maiman (1927-2007) at Hughes Research Laboratories. 'The laser consisted of a high-voltage helical flashlamp surrounding a small tubular synthetic ruby crystal coated with silver on its two end faces, with a small scratch etched into the emitting face. Maiman measured the width of the spectral line emitted in millisecond pulses of coherent 694.3-nm deep red light, and the ruby laser was born, mankind's first creation of coherent light. Shortly afterwards, the helium-neon (HeNe) laser (632.8 $\mathrm{nm}$ ) and neodymium:yttrium aluminum garnet (Nd:YAG) laser (1064 nm) were developed in 1961, followed by the argon laser (488 nm, $514.5 \mathrm{~nm}$ ) and near-infrared (nearIR) diode laser in 1962, the carbon dioxide (CO2) laser in
$1964(10,600 \mathrm{~nm})$, the tunable pulsed dye laser in 1969 $(580-595 \mathrm{~nm})$, and the excimer laser in 1970 (172 nm). ${ }^{2}$

\section{MILESTONES IN LASER MEDICINE AND SURGERY}

Laser medicine and surgery was pioneered by Dr. Leon Goldman (1906-1997), an American dermatologist and surgeon. Goldman studied the interaction of laser light with biological systems, including early clinical studies on humans. In 1961, Goldman became the first researcher to use a laser to treat a human skin disease, melanoma. In 1966, Goldman supervised the first laser operation to remove a tumor without hemorrhage. Goldman was one of the founders of the American Society for Laser Medicine and Surgery (ASLMS) in 1979, and in 1981, he was the first ASLMS president.

From the 1980s, more powerful and compact lasers became available for use in medicine and surgery: $\mathrm{CO}_{2}$ lasers were used for incision and excision of tissues; argon lasers were used in ophthalmic surgery and dermatology; $\mathrm{Nd}$ :YAG lasers, without and with frequency doubling using a potassium titanyl phosphate crystal, were used for dermatologic and laparoscopic surgery. These secondgeneration lasers were all designed based on continuous wave delivery, and thus, they were limited to inducing non-selective photothermal injury causing unwanted complications. Accordingly, they required a long learning curve and proper use by experienced laser surgeons. Nonetheless, many breakthrough results were achieved with these systems by laser clinicians who understood the finer intricacies of laser/tissue interactions.

The next most significant clinical advancement in laser therapy was achieved with application of the principle proposed by Dr. Richard Rox Anderson (1960-) and John A. Parrish in 1983, the theory of selective photothermolysis, which mandated the use of pigment-specific, shortpulsed lasers. Doing so facilitated histologically selective and highly contained thermal injury for the treatment of vascular and pigmented lesions, with minimal, or even without, scarring. In Anderson and Parish's study, pigmented structures, cells, and organelles were irradiated in vivo with suitably brief pulses to cause selective damage to the pigmented targets while sparing the surrounding normal tissues. Precise aiming was not required, because inherent optical and thermal properties provided target selectivity. Selective damage to cutaneous microvessels and to melanosomes within melanocytes could be consistently observed after radiation. The first laser to fully exploit the theory was the yellow pulsed dye laser to treat port wine stains. ${ }^{3}$ This principle was 
continuously developed further and was followed by the first Q-switched laser for tattoo and pigment removal: Qswitching enabled lasers to deliver even shorter pulse widths in the nanosecond domain. The shorter the pulse width, the higher the selectivity and the more contained the damage was within the target pigment.

Another major advancement was the introduction of "scanning" technology in the early 1990s for $\mathrm{CO}_{2}$ and Er:YAG lasers, enabling precise computerized control of laser beams. Scanning was then combined with fractionation of the laser beam into myriad microbeams, leaving areas of untreated tissue between microcolumns of injured tissue. Upon combining skin cooling with the laser in clinical practice, however, researchers found that the lower skin temperatures associated with the use of cooling caused a decrease in the diameters and the extent of intradermal thermal injury of what became called "microscopic treatment zones (MTZs)": it was believed that this could interfere with achieving consistent treatment results. Therefore, a need for new advanced technology that could ensure computerized control of appropriate laser energy densities and pulse widths that are adjusted automatically according to temperature changes in the skin surface arose so that MTZs are created in a uniform and consistent manner. ${ }^{4}$ Recently, one laser manufacturer resolved this challenge by incorporating their patented technology called "dynamic thermal optimization (DTO)" into a commercialized product that measures the temperature of the skin and automatically changes the energy density and pulse width to ensure the laser is always tuned to desired treatment parameters.

These recent advances in laser pulsing, scanning, and fractionation technologies have helped revolutionize the clinical applications of laser medicine and surgery. Now, consistent, clinical results can be obtained with less complications and shorter patient downtime, as well as increased public awareness of the availability of these new advances in laser technology. The growth of the medical laser industry is expected to grow exponentially, and according to the latest report by Grand View Research, the global medical laser market is expected to reach USD 12.5 billion by $2022 .^{5}$

\section{THE FUTURE}

Lasers continue to find ever-growing applications in medicine and surgery, and research is being driven by multilateral collaborations among practitioners and innovators in industry, science, and medicine. Recently, the use of lasers in both the near and mid-infrared range is being tried by many scientists and physicians to measure blood glucose levels in both a harmless and non-invasive way. Diabetes is a disease that affects millions of people who suffer from the daily stress of having to measure their blood glucose levels, which involves not only some pain but also the risk of infection. New technology using lasers as a measurement tool could potentially allow for pain-free and low-cost blood glucose measurement. However, the results from trials suggest that accurate measurement with a minimal margin of error will be a challenge for this new application. Nevertheless, a start has been made.

Ophthalmology is also a clinical area wherein lasers are predominantly used. Although intense pulsed light (IPL) is strictly speaking not a laser, many ophthalmologists are trying this technology for treatment of dry eye syndrome. The public is closely paying attention to this new IPL approach, since IPL was originally developed for dermatologic procedures, such as reduction of pigmented and vascular lesions or unwanted hairs. Applying IPL from canthus to canthus is believed to stimulate the Meibomian glands to return to their normal function. As a result, a dry lipid layer will return to a natural flow of lipids, thereby reducing evaporation of tears. The clinical efficacy of this approach was demonstrated and published in 2015 in Photomedicine and Laser Surgery. ${ }^{6}$ Another new approach that is expected to be commercially available soon is an advanced approach to treat diabetic macular edema using a new laser that involves specifically targeting the retinal pigment epithelium, without damaging the overlying retinal layers, to induce repair by cell migration and proliferation using a 527-nm highly pigment-selective laser. In a study published in 2015 Graefe's Archive for Clinical and Experimental Ophthalmology, researchers noted significant improvement in best corrected visual acuity.

In the field of dentistry, one laser manufacturer has introduced and commercialized shock wave enhanced emission photoacoustic streaming (SWEEPS). The technology uses Er:YAG laser to create non-thermal photoacoustic shock waves within cleaning and debriding solutions introduced in the dental canal. According to a paper published in 2017 by the Journal of the Laser and Health Academy, the technology delivers a precise concentration of shock waves into the cleaning fluids, reaching deep into the lateral canals and microscopic tubules to remove tissue, debris, biofilm, and bacteria. Thus, it promises to significantly enhance the efficacy of standard photoninduced photoacoustic streaming laser-induced irrigation procedures. $^{8}$ 
Lastly, in the dermatology field, one of the latest innovations is a new technology that utilizes the production of ultrasonic pulses while transmitting light through direct contact with the skin. This new technology, named "sonoillumination," was presented by Paul J.D. Whiteside at the annual conference of the 2017 American Society for Laser Medicine and Surgery. According to Whiteside, the new technology uses ultrasonic pulsation in conjunction with contact-based transmission from a laser to alter the properties of skin tissue during the procedure. The laser will not work unless it is actually in contact with the patient's skin. ${ }^{9}$ This is could help to reduce safety concerns regarding potential eye damage during laser procedures, as well as to improve the precision and transmission of laser through the surface layers of the skin. The future of the laser in all aspects of medicine and surgery continues to grow brighter and brighter.

\section{REFERENCES}

1. Mainman TH. Stimulated optical radiation in ruby. Nature 1960;187:493-4.

2. Calderhead RG. Photobiological basics of photosurgery and phototherapy. 1st ed. Seoul, Korea: Hanmi Medical Publishing Co.; 2011. p.4-5.
3. Anderson RR, Parrish JA. Selective photothermolysis: precise microsurgery by selective absorption of pulsed radiation. Science 1983;220:524-7.

4. Laubach H, Chan HH, Rius F, Anderson RR, Manstein D. Effects of skin temperature on lesion size in fractional photothermolysis. Lasers Surg Med 2007;39:14-8.

5. Medical laser systems market analysis [Internet]. San Francisco, CA, US: Grand View Research, Inc.; 2016. [cited 2016 Mar]. Available from: http://www.grandviewresearch. $\mathrm{com} /$.

6. Toyos R, McGill W, Briscoe D. Intense pulsed light treatment for dry eye disease due to meibomian gland dysfunction; a 3-year retrospective study. Photomed Laser Surg 2015;33:41-6.

7. Park YG, Kim JR, Kang S, Seifert E, Theisen-Kunde D, Brinkmann $R$, et al. Safety and efficacy of selective retina therapy (SRT) for the treatment of diabetic macular edema in Korean patients. Graefes Arch Clin Exp Ophthalmol 2016;254:1703-13.

8. Lukac N, Muc BT, Jezersek M, Lukac M. Photoacoustic endodontics using the novel SWEEPS Er:YAG laser modality. J Laser Health Acad. In press 2017.

9. Whiteside PJD, Qian C, Golda N, Hunt HK. Ultrasonic modulation of tissue optical properties in ex vivo porcine skin to improve transmitted transdermal laser intensity. Lasers Surg Med. In press 2017. 